\title{
Seasonal and Daily Patterns of the Mating Calls of the Oyster Toadfish, Opsanus tau
}

\author{
JACEY C. VAN WERT ${ }^{1, *}$ AND ALLEN F. MENSINGER ${ }^{1,2}$ \\ ${ }^{I}$ Marine Biological Laboratory, 7 MBL Street, Woods Hole, Massachusetts 02543; \\ and ${ }^{2}$ Department of Biology, University of Minnesota Duluth, 1035 Kirby Drive, \\ Duluth, Minnesota 55812
}

\begin{abstract}
Acoustic communication is vital across many taxa for mating behavior, defense, and social interactions. Male oyster toadfish, Opsanus tau, produce courtship calls, or "boatwhistles," characterized by an initial broadband segment (30-50 ms) and a longer tone-like second part (200-650 ms) during mating season. Male calls were monitored continuously with an in situ SoundTrap hydrophone that was deployed in Eel Pond, Woods Hole, Massachusetts, during the 2015 mating season. At least 10 vocalizing males were positively identified by their unique acoustic signatures. This resident population was tracked throughout the season, with several individuals tracked for extended periods of time (72 hours). Toadfish began calling in mid-May when water temperature reached $14.6^{\circ} \mathrm{C}$ with these early-season "precursor" boatwhistles that were shorter in duration and contained less distinct tonal segments compared to calls later in the season. The resident toadfish stopped calling in mid-August, when water temperature was about $25.5^{\circ} \mathrm{C}$. The pulse repetition rate of the tonal part of the call was significantly related to ambient water temperature during both shortterm (hourly) and long-term (weekly) monitoring. This was the first study to monitor individuals in the same population of oyster toadfish in situ continuously throughout the mating season.
\end{abstract}

\section{Introduction}

Communication reflects advanced social behavior and allows conspecifics to recognize one another. The spectral and temporal parts of acoustic communication encode informa-

Received 25 April 2018; Accepted 20 November 2018; Published online 8 February 2019.

* To whom correspondence should be addressed. Email: jcvanwert@ ucsb.edu.

Abbreviations: CV, coefficient of variation; MBL, Marine Biological Laboratory; NOAA, National Oceanic and Atmospheric Administration; NWS, National Weather Service; PRR, pulse repetition rate.

Online enhancement: audio file. tion that allows for species recognition, defense, and courtship (Beecher, 1989; Wilkins et al., 2013). Although terrestrial acoustic signaling is well studied and marine mammal bioacoustics is a growing field, much less is known about acoustic communication in fishes (Amorim, 2006). However, several fish species have become model systems for bioacoustics because of their sound-producing ability. Among them are batrachoidid fishes (toadfish and midshipman), which possess sexually dimorphic sonic muscles that surround the swim bladder and contract to produce vocalizations (Skoglund, 1961; reviewed by Bass and McKibben, 2003; Amorim, 2006). The oyster toadfish, Opsanus tau (Linnaeus, 1766), produces characteristic vocalizations, including territorial, agonistic, and, in the case of males, advertisement calls (Edds-Walton et al., 2002; Maruska and Mensinger, 2009).

In mid-spring, sexually mature male $O$. tau individuals establish nest sites under hard substrate and produce advertisement calls, or "boatwhistles" (Gray and Winn, 1961), through late summer. Female fish are attracted by the calls and will deposit eggs on the upper surface of the nest for fertilization (Fish, 1972). Males are polygynous and may continue to attract other females, while guarding the nests until the larval fish detach from the nest (Lowe, 1975; Mensinger et al., 2003).

The boatwhistle of each male has a unique acoustic signature (Fish, 1972; Fine, 1983; Edds-Walton et al., 2002; Putland et al., 2018) that consists of a brief high-amplitude broadband grunt $(\sim 30-50 \mathrm{~ms})$ followed by a regular pulsing period ( 200-650 ms) (Tavolga, 1958; Edds-Walton et al., 2002). Each boatwhistle is characterized by differences in waveform, sound duration, and energy distribution in harmonics that allow for identification of individuals (Amorim and Vasconcelos, 2008; Putland et al., 2018). In addition, the second portion has a distinct pulse repetition rate (PRR; fundamental frequency) that ranges from 90 to $250 \mathrm{~Hz}$ (Winn, 1972; Edds-Walton et al., 2002; Mensinger, 2014) and is hypothesized to influence fe- 
male mate choice (Edds-Walton et al., 2002). Opsanus tau can detect low-frequency sound with its inner-ear organs and lateral line, both of which are sensitive to particle motion between 50 and $300 \mathrm{~Hz}$ (Fay and Edds-Walton, 1997; Maruska and Mensinger, 2015) and between 80 and $300 \mathrm{~Hz}$ (Radford and Mensinger, 2014), respectively.

Toadfish vocalizations have been studied in an ecological context, and they indicate a close link to their environment. Amorim et al. (2006) measured seasonal variations of sound production by the Lusitanian toadfish, Halobatrachus didactylus, and found that in response to rising water temperatures, boatwhistle call rate, duration, and fundamental frequency increased and pulse period decreased. Along the eastern seaboard of the United States, Fine (1978) recorded toadfish boatwhistle calls beginning in May when water temperature was $15.7{ }^{\circ} \mathrm{C}$ and lasting through October with temperature effects observed on PRR but not on call duration and amplitude. Recent studies using passive acoustic monitoring to record large populations of toadfish also found a strong correlation between PRR and water temperature (Ricci et al., 2017). The variation in structure, durations, and frequencies of toadfish vocalizations (Fine, 1978; Edds-Walton et al., 2002) suggests a complex acoustic system (Maruska and Mensinger, 2009).

However, most previous call monitoring has been intermittent, with weekly sampling in different areas or using signal detection software to monitor large populations; thus, recording from individual oyster toadfish for extended, consecutive time periods has not been reported. It has recently been discovered that oyster toadfish have reestablished residence in Eel Pond, located next to the Marine Biological Laboratory (MBL), in Woods Hole, Massachusetts (Putland et al., 2018). Several resident toadfish were within detection range of a hydrophone deployed at the MBL dock. This relatively small number of toadfish provided a unique opportunity to continuously monitor their vocalizing patterns throughout the mating season, which could otherwise be difficult to detect from wild populations with greater densities. The goals of this study were to monitor the vocalizations of male oyster toadfish (1) individually over a period of days and (2) as a population across the entire season, in their natural environment in Woods Hole, and (3) to determine how toadfish calling parameters (PRR and duration) were influenced by water temperature. Based on previous studies, it was predicted that toadfish calling rates would peak during the mating season and that temperature would impact boatwhistle PRR but not duration.

\section{Materials and Methods}

Study site

Oyster toadfish Opsanus tau L. (Linnaeus, 1766) vocalizations were recorded in situ from beneath the floating Marine Resources Center dock at MBL in Eel Pond, Woods Hole $\left(41^{\circ} 31^{\prime} 32.22^{\prime \prime} \mathrm{N}, 70^{\circ} 40^{\prime} 16.74^{\prime \prime} \mathrm{W}\right)$. The pond bottom consists primarily of soft sediments interspersed with rocky substrate and man-made detritus. The pond is directly connected to Great Harbor through a narrow canal and is subject to mixed semidiurnal tidal cycles. Dock access is open to the public Monday through Friday from 0730 to 1700 . A drawbridge restricts large-boat egress to the pond during the night from 1900 to 0700 (May 15-June 14) or from 2100 to 0600 (June 15September 15).

\section{Field recordings}

A hydrophone (SoundTrap300, Ocean Instruments, Warkworth, Auckland, New Zealand) was suspended from the dock crossbeams below the surface planks via a polyester rope, with the top of the hydrophone maintained $1.2 \mathrm{~m}$ beneath the water surface and its bottom ranging from 1.2 to $2.2 \mathrm{~m}$ above the substrate, depending on tidal conditions. A brick was attached at the end of the rope to serve as an anchor and to minimize hydrophone movement. The SoundTrap was deployed May 18 through August 19, 2015, and it recorded continuously (24 h $\mathrm{d}^{-1}$, minimal sampling rate $24 \mathrm{kHz}$ ), with the exception of 2- to 4-h intervals about twice per week between 1200 and 1600 for data download and battery recharge. An occasional longer interruption was encountered due to environmental conditions or battery issues; however, $92 \%$ of the experimental window was sampled. The SoundTrap was also deployed in the same location June 23-August 2, 2016, and an additional $41 \mathrm{~h}$ of data from summer 2016 (July 8- July 14) were used to measure the PRR of 6 distinctive toadfish boatwhistles over $5 \mathrm{~h}$ and 1 distinctive toadfish boatwhistle over $36 \mathrm{~h}$.

Temperature and light intensity were logged every minute using a HOBO Pendant (Onset Computer, Bourne, MA) attached to the recording SoundTrap. High- and low-tide heights were found at National Oceanic and Atmospheric Administration (NOAA) Tides and Currents (station 8447930, 41 ${ }^{\circ} 31.4^{\prime} \mathrm{N}$, $70^{\circ} 40.3^{\prime}$ W) (Tides and Currents, 2015). Daily sunset times and lunar cycles were obtained from NOAA National Weather Service (NWS) for Falmouth, Massachusetts (NWS, 2015).

\section{Sound file analysis}

Sound files were analyzed offline using Raven Pro 1.4 for Windows (Bioacoustics Research Program, 2014) and MATLAB software (MathWorks, 2018). All data were band-pass filtered between 50 and $4000 \mathrm{~Hz}$ to exclude low- and highfrequency sound, respectively. Toadfish calls were identified by listening to sound files and examining the oscillograms and spectrograms (1024-point fast Fourier transform; sampling frequency $24-96 \mathrm{kHz}$; Hamming window). Boatwhistle call rate, duration, and PRR were analyzed. The PRR was calculated by counting the first 15-20 contiguous pulses with approximately equal amplitudes of the second (tonal) segment of each call and dividing by the duration of these pulses (Fig. A1).

Toadfish grunts and boatwhistles were recorded; however, only boatwhistles were analyzed. Grunts could be differentiated 
from boatwhistles because they were often short ( $<100 \mathrm{~ms})$ and lacked a distinct tonal segment. A vocalization was classified as a boatwhistle if it had a minimum duration of $100 \mathrm{~ms}$ and an initial grunt component followed by a distinct tonal segment (Tavolga, 1958; Maruska and Mensinger, 2009). To track and differentiate individual males, we used boatwhistle call characteristics, including spectrogram composition, waveform shape, calling interval, and relative amplitude (Fig. 1; Audio S1, available online). Spectral consistency in the call and high site fidelity exhibited by the males (Fish, 1972; Fine, 1983; EddsWalton et al., 2002; Putland et al., 2018) allowed individuals to be tracked with a high degree of confidence for extended periods (72 hours).

\section{Resident and individual call rate}

Preliminary analysis indicated that boatwhistle production was consistently high around sunset, and, therefore, the number of calls were summarized between 30 minutes before and after sunset. The number of boatwhistles was summed daily during this hour-long window from May 22 to August 17, 2015, and was used to calculate the resident toadfish boatwhistle calling rate. To determine individual calling rates, toadfish $(n=5)$ with distinct boatwhistles were tracked over a 72-hour period from June 29 to July 2, 2015. The number of boatwhistles produced by each toadfish for the first 10 minutes of each hour was quantified. If anthropogenic or environmental sound precluded analysis during these time frames, the first 10-minute window after the external sound subsided was analyzed.

\section{Call duration and pulse repetition rate}

To address seasonal variation, we examined toadfish $(n=$ 1-6) for waveform characteristics (PRR and duration) weekly, with the first 15 calls initiated after midnight (between 0000 and 0130) from each distinguishable individual analyzed. This midnight time window coincided with high calling activity, it reduced potential variations due to changing sunrise and sunset times, and it assured that anthropogenic sound (boat and dock foot traffic) was at minimum, to allow accurate characteristics of the waveforms. While this time window provided the highest fidelity signals due to low background noise, it precluded the ability to state with $100 \%$ confidence that the same individual was recorded on consecutive nights.

To examine individual changes in the PRR across shorter time periods, boatwhistles from individuals were tracked for 5 hours ( $n=6$ fish; all boatwhistles) and 36 hours ( $n=1$ fish; first 5 boatwhistles per hour) and compared to changes in temperature.

\section{Statistical analysis}

All statistical analyses were run in RStudio (RStudio Team, 2018). All data were tested for normality using the ShapiroWilk normality test. To determine whether resident toadfish boatwhistle calling rates changed between the 30 minutes before and after sunset windows, a Wilcoxon signed rank test was used. Linear regressions were used to assess whether there was a relationship between water temperature and mean boatwhistle PRR, as well as between water temperature and mean boatwhistle duration, throughout the season. However, repeated measures of individual specific calls could not be accounted for given the experimental design, and the coefficients of variation were therefore reported with these results. Thus, the parameter estimates of linear regression contain unexplained variance and must be considered when evaluating the results. Boatwhistle parameters were calculated during peak calling season (June 5-July 13, 2015) because this time interval pro-

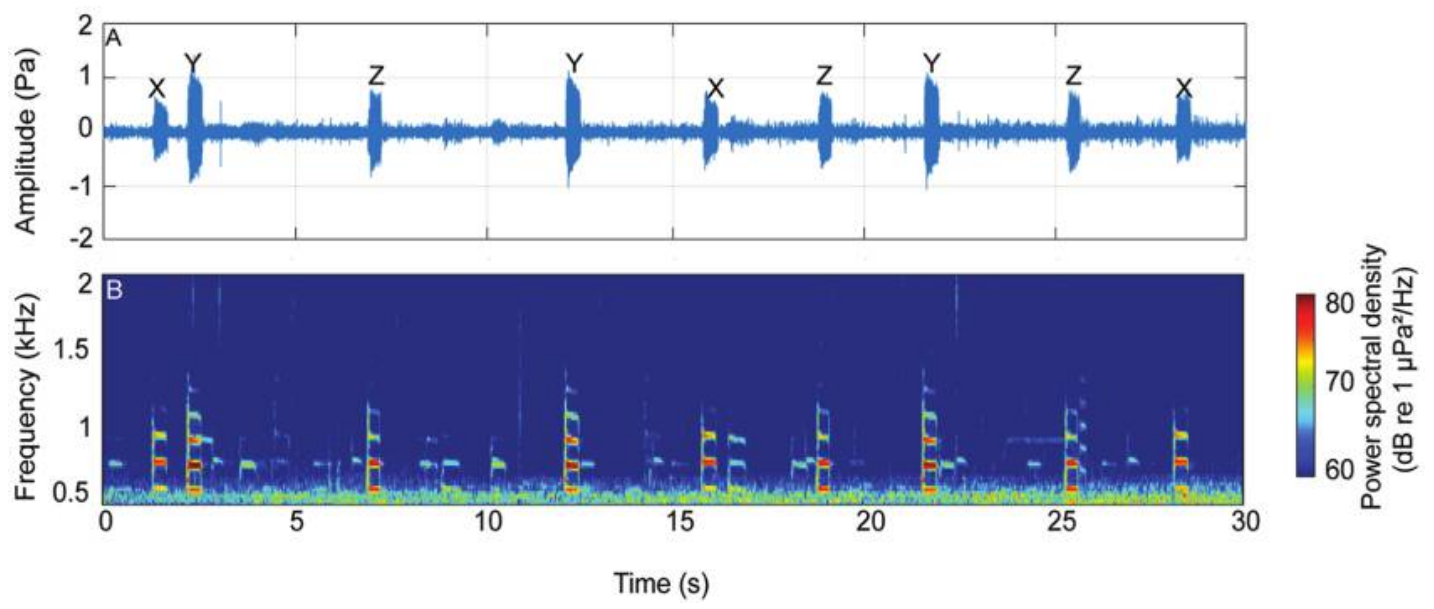

Figure 1. Toadfish alternate boatwhistle calling. Toadfish boatwhistles are shown during a 30-second calling window. Three distinct individuals were identified $(\mathrm{X}, \mathrm{Y}, \mathrm{Z})$ based on waveform shape, spectrogram components, and relative amplitude. (A) Oscillogram and (B) spectrogram view; spectrograms were produced using fast Fourier transform $($ FFT $)=2048$ points, 80\% overlap, and a Hamming window. 
vided a sufficient number of calls with a low signal-to-noise ratio and distinguishable start and end of calls for PRR calculations. To measure the relationship between water temperature and the boatwhistle PRR over 5 hours and 36 hours, a linear regression analysis and Spearman rank order correlation test were used, respectively.

\section{Results}

The SoundTrap successfully recorded both toadfish vocalizations and anthropogenic sound. Transient outboard and inboard motorboat sound was recorded throughout the drawbridge operating hours, with a sharp decrease in anthropogenic sound between 0000 and 0530 each night. About six individual toadfish were routinely identified each night during the peak calling period, and several could be tracked for days at a time. Sounds were also detected from up to 4 other individuals $(n=$ 10 total); however, based on lower call amplitudes, it appeared that these fish were farther away from the hydrophone, and the signal-to-noise ratio was usually insufficient to accurately quantify call parameters.

The SoundTrap was deployed on May 18 (water temperature $12.9^{\circ} \mathrm{C}$ ), and boatwhistle calls were first detected on May 22 (water temperature $14.6{ }^{\circ} \mathrm{C}$ ). The last boatwhistle vocalization was recorded on August 17 (water temperature $25.5^{\circ} \mathrm{C}$ ), and the SoundTrap was removed for the season on August 19 (water temperature $26.2^{\circ} \mathrm{C}$ ). Water temperature ranged from 12.8 to $28.5^{\circ} \mathrm{C}$ during the 2015 deployment, with a maximum range of daily water temperature change of $2{ }^{\circ} \mathrm{C}$ and with higher temperatures often encountered in the afternoon.
The number of boatwhistles detected in the hour block surrounding sunset was used to determine the calling rate of the resident toadfish in Eel Pond in 2015. There was a significant increase in calling activity in the 30-minute window after sunset compared to the 30 minutes prior to sunset (Wilcoxon signed rank test: $Z=-6.31 ; P<0.001)$. The resident calling rate was relatively low from May 18 to June 5 and after July 17, with often $<20$ boatwhistles per hour. The calling rate increased mid-June, reaching a maximum of 703 boatwhistles per hour on June 19 (water temperature $18.5^{\circ} \mathrm{C}$ ), with most dates between June 5 and July 18 showing at least 250 boatwhistles per hour during twilight (Fig. 2). Although full moons appeared on June 3 and July 1 and new moons occurred on June 17 and July 15, correlating call number with lunar and/ or tide changes was tenuous at best because only one full moon and two new moon events transpired during peak calling season, and equipment outages also occurred near these times.

The spectral properties of boatwhistles changed during the calling season and became more fine-tuned, with clearer separation of harmonics as the season progressed (Fig. 3). In the beginning of the season (water temperature $14.5^{\circ} \mathrm{C}$ ), boatwhistle calls lacked periodicity, or regularity in waveform structure, in the tonal segment (Fig. 3A). These "precursor" calls were relatively short (100-350 ms), and the grunt and tonal portions were not well defined. As the season progressed, the distinction between the broadband grunt and the succeeding tonal segment became more defined, with the second portion of boatwhistle more tonal and with individuals more easily identified based on waveform and spectral properties (Fig. 3B-D).

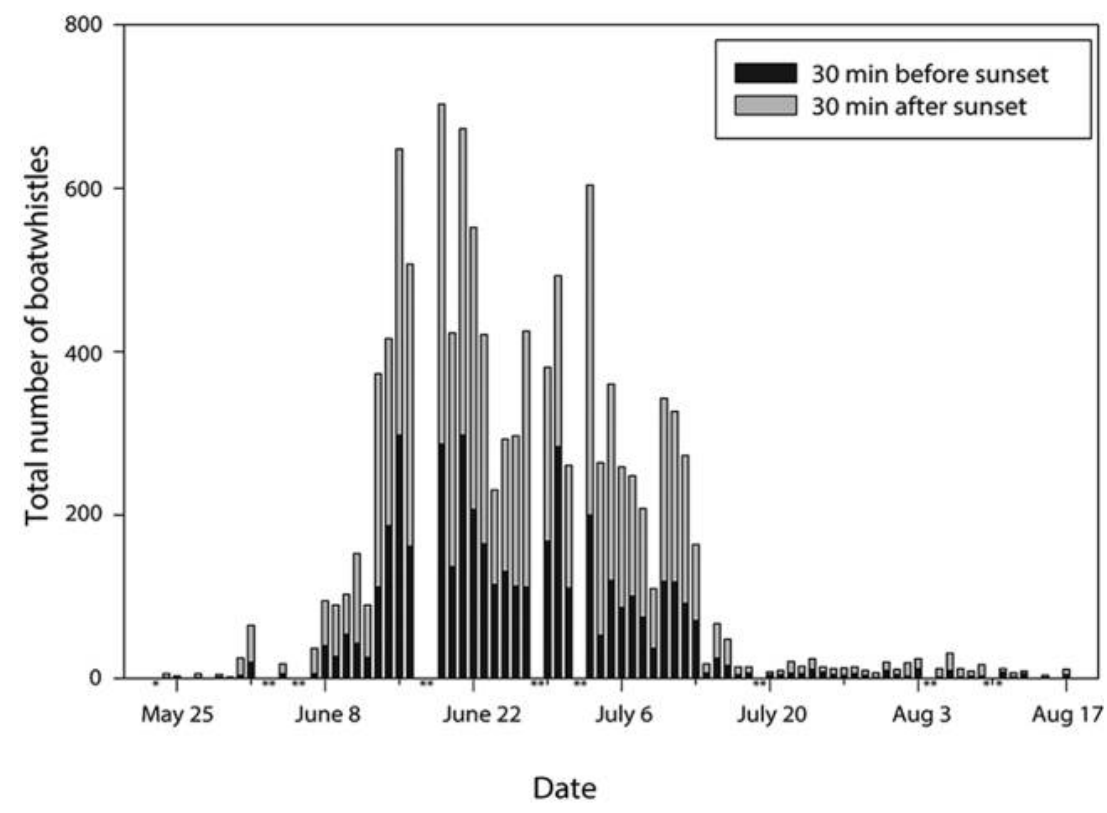

Figure 2. Toadfish population calling rate. Total number of calls in the 30 minutes before (black) and 30 minutes after (gray) sunset are plotted versus the date (May 22-August 17, 2015). Asterisks along the $x$-axis indicate dates that recordings were unavailable. 
A. May $2414.5^{\circ} \mathrm{C}$

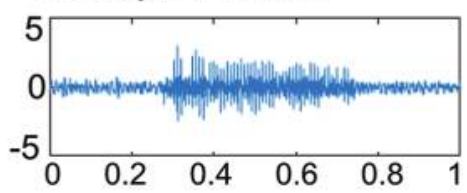

B. May $2915.0^{\circ} \mathrm{C}$

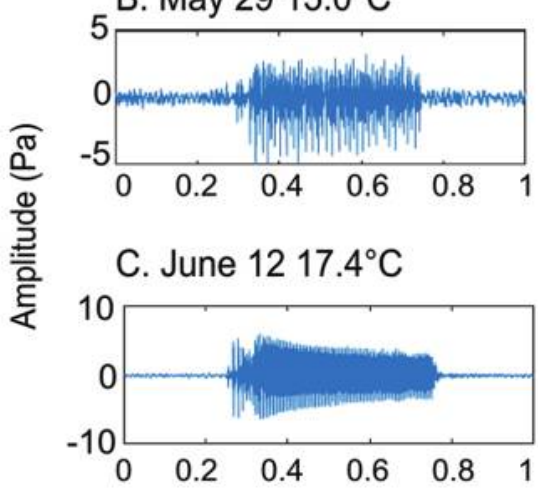

D. August $724.2^{\circ} \mathrm{C}$

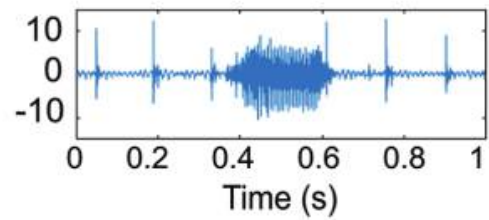

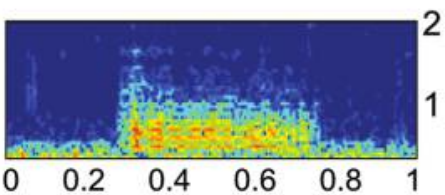
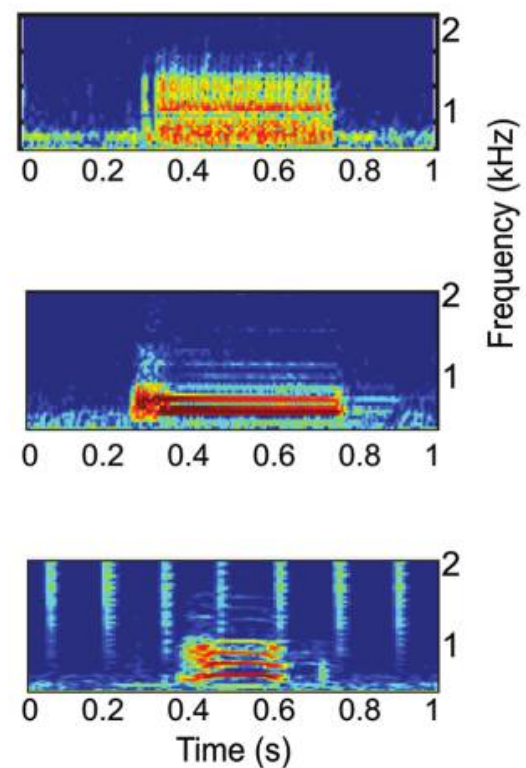

Power spectral density (dB re $1 \mu \mathrm{Pa}^{2} / \mathrm{Hz}$ )

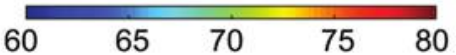

Figure 3. Toadfish boatwhistles. Oscillograms (left) and spectrograms (right) of toadfish boatwhistles recorded throughout the mating season are plotted versus time. (A) May 24, (B) May 29, (C) June 12, (D) August 7, 2015. Spectrograms were produced using fast Fourier transform $($ FFT $)=2048$ points, $80 \%$ overlap, and a Hamming window. Note the different amplitude scales.

Boatwhistle duration and PRR were analyzed throughout the peak calling season from June 5 up to August 7, during which time corresponding water temperature rose from 15.4 to $24.2^{\circ} \mathrm{C}$ (Fig. 4A). The mean call duration ranged from 103 to $1052 \mathrm{~ms}$ throughout the season (Fig. 4B) and was found to be inversely related to increased temperature $\left(R^{2}=0.45 ; F_{1,60}=50.2, P<\right.$ $0.001)$. The coefficients of variation $(\mathrm{CV}$; standard deviation per mean) between individual mean duration of calls within each day varied from $9.1 \%$ to $50.2 \%$, and temperature had no effect on CV. The mean PRR ranged from 132 to $223 \mathrm{~Hz}$ from June 5 to July 13, 2015 (Fig. 4C), and showed a strong, positive relationship with water temperature $\left(R^{2}=0.90 ; F_{1,48}=493.1\right.$, $P<0.001)$. The $\mathrm{CV}$ between individual mean PRR of calls within each day varied from $0.3 \%$ to $4.1 \%$, and again temperature had no effect on CV.

Individual toadfish calls were also tracked. The mean PRR of 1 individual toadfish varied throughout a 36-hour time interval by $11 \mathrm{~Hz}$, with temperature deviating by $1.2{ }^{\circ} \mathrm{C}$ during this interval (Fig. 5). There was a significant positive relation- ship between water temperature and mean boatwhistle PRR for this individual toadfish $\left(n=1, r_{\mathrm{s}}=0.728, P<0.0001\right)$. The mean PRR of multiple toadfish $(n=6)$ across a 5-hour interval varied by $6 \mathrm{~Hz}$, during which time the temperature decreased from 20.47 to $20.10{ }^{\circ} \mathrm{C}$ (Fig. 6). A linear regression was plotted with the mean PRR of all toadfish $(n=2298$ calls), and analysis showed a positive, significant trend $\left(R^{2}=\right.$ 0.85 , df $=6, P<0.01$ ).

Calling rates varied considerably over the time of day and across individuals. The calling rates of 5 individual males were tracked over a 72-hour period (Fig. 7), with less than $1 \%$ boatwhistle call overlap observed ( $n=2428$ calls). Toadfish B dominated the time period, with $53 \%$ of the total calls $(n=$ 1287), followed by toadfish A, which generated 570 calls ( $23.4 \%$ of the total). Toadfish C, D, and E calling rates were similar but less frequent, with call number ranging from $170(7.0 \%)$ to $209(8.6 \%)$. Calling was relatively infrequent during the day, with declines seen at sunrise and minimal calling at 0700 (3.6 \pm 5.4 boatwhistles per hour) that gradually increased at sunset 
A

Month

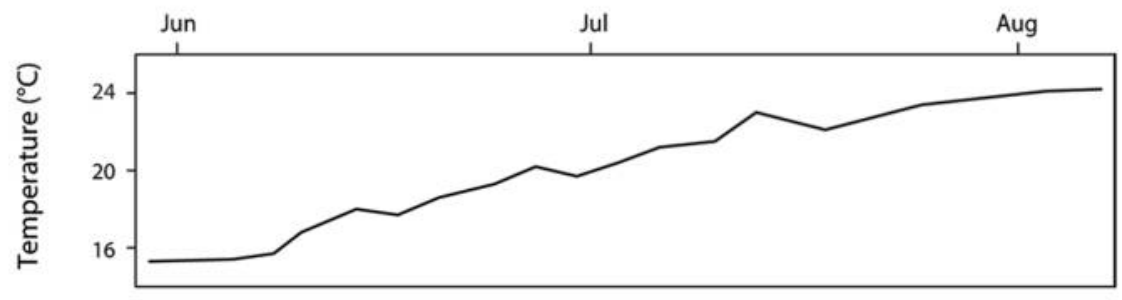

B

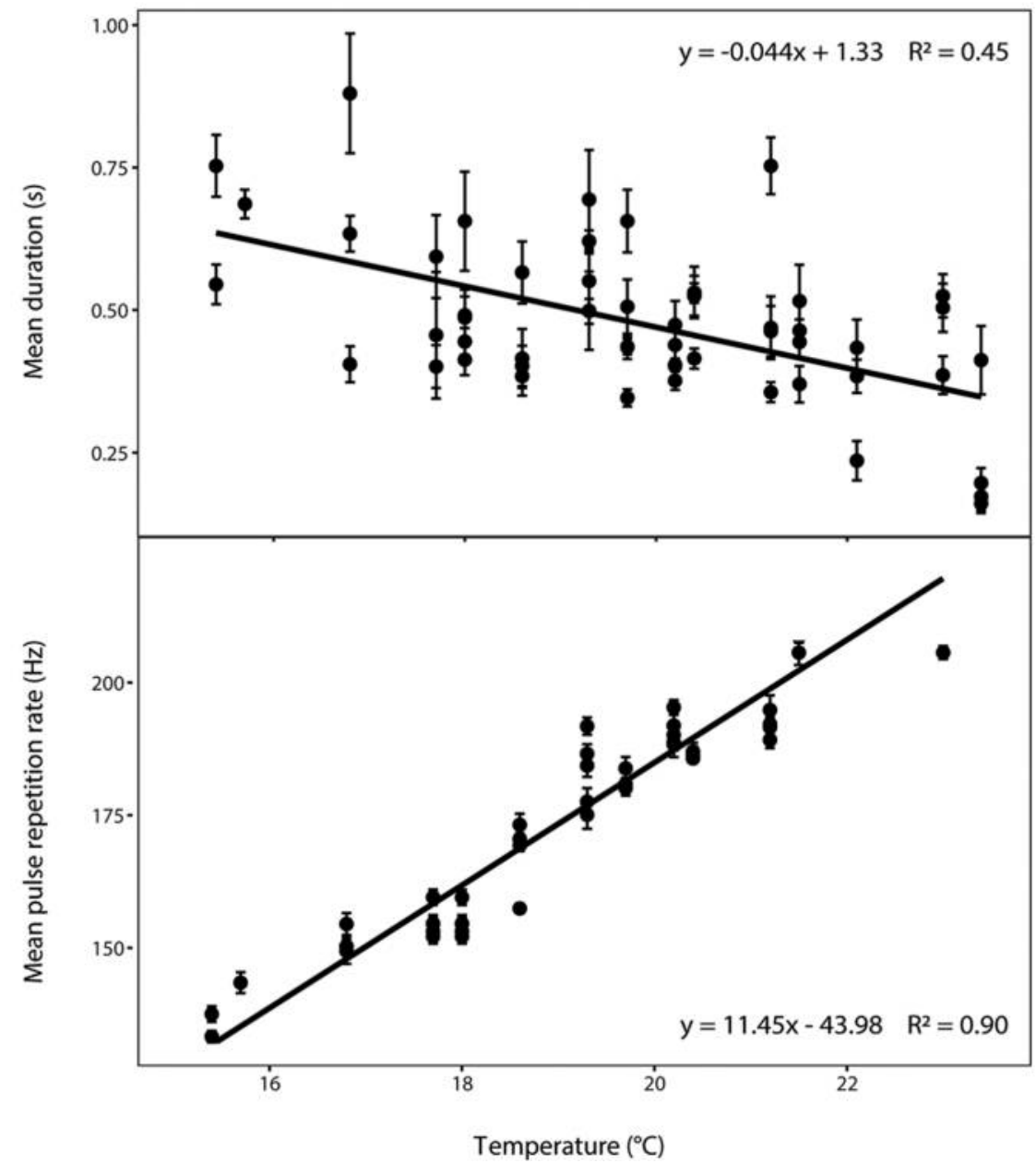

Figure 4. Effect of temperature on boatwhistle duration and pulse repetition rate (PRR). (A) Ambient midnight water temperature is plotted for each selected date throughout the mating season (June 5-August 7, 2015). (B) Mean boatwhistle duration (sampling period: June 5-August 7, 2015) or (C) PRR (sampling period: May 30-July 13, 2015 ) is plotted versus ambient water temperature. Each data point represents the mean boatwhistle (B) duration or (C) PRR for the first 15 calls after midnight from individual toadfish $(n=62)$. Note that sampling methods resulted in repeated measures. Error bars $=1 \mathrm{SD}$.

among all fish. Calling rates for all 5 individuals were greatest between 1900 and 0200 and varied from a maximum of 144 boatwhistles per hour to 432 boatwhistles per hour.

\section{Discussion}

The SoundTrap successfully detected oyster toadfish vocalizations along with other anthropogenic and biological sounds near the MBL dock in Eel Pond. About 10 different male toadfish were identified by their acoustic signatures. Several individuals were tracked over a period of days, and the resident population was tracked throughout the entire season. Resident toadfish began calling in mid-May at water temperatures of $14.5^{\circ} \mathrm{C}$, peaked from mid-June to early July, and then declined substantially from mid-July until the SoundTrap was removed in late August. This site contains one of the most northern pop- 


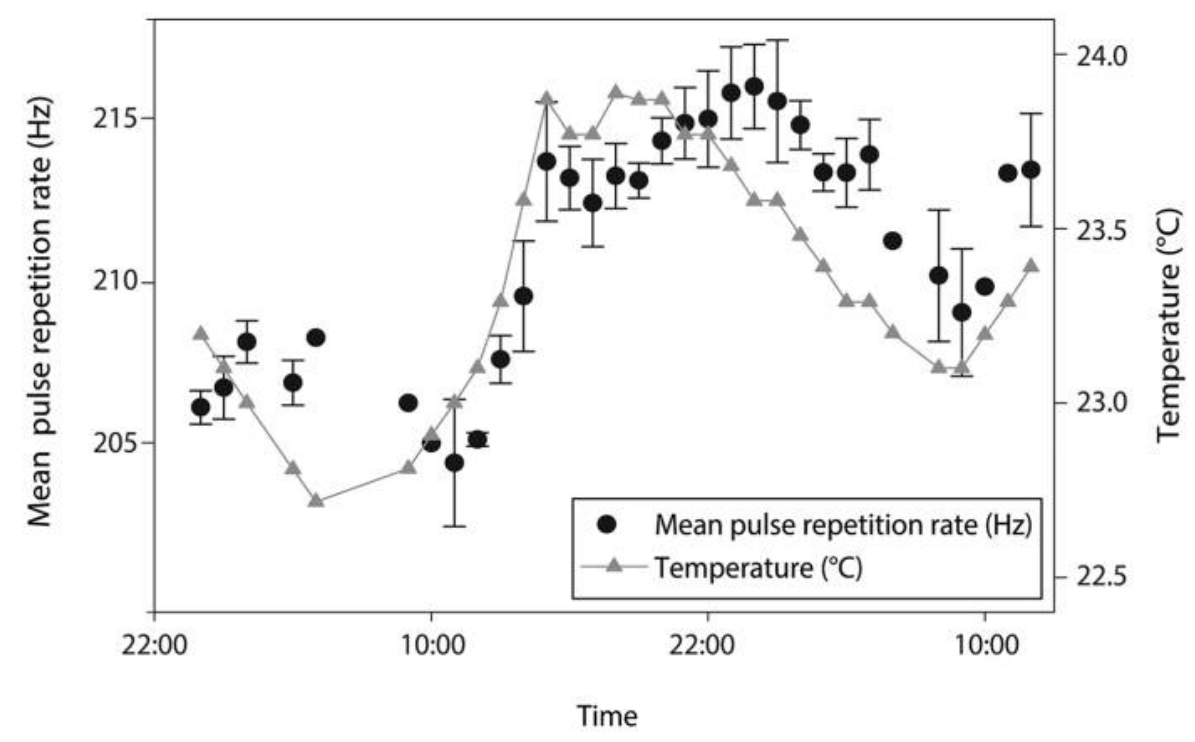

Figure 5. Individual variation in toadfish boatwhistle. The mean pulse repetition rate (PRR) (first 5 boatwhistles per hour) is plotted versus time for a single toadfish across 36 hours (0000 July 13-1200 July 14, 2016). Error bars = $1 \mathrm{SD}$.

ulations of Opsanus tau; and calling was initiated later than most southern populations, presumably due to colder springtime water temperatures (Fine, 1978; Edds-Walton et al., 2002).

Toadfish were recently detected in Eel Pond after an absence of at least 20 years. Whether these fish migrated into the pond or were released or escaped from the adjacent Marine Resources Center of the MBL is unknown; however, about
10 males have established breeding sites within range of the hydrophone. The first confirmed juvenile toadfish (year 0) was detected in the summer of 2018 , indicating that a breeding population had been established.

Several previous studies monitored oyster toadfish vocalizations; however, most were intermittent and unable to monitor individual fish for extended periods of time (Fine 1978;

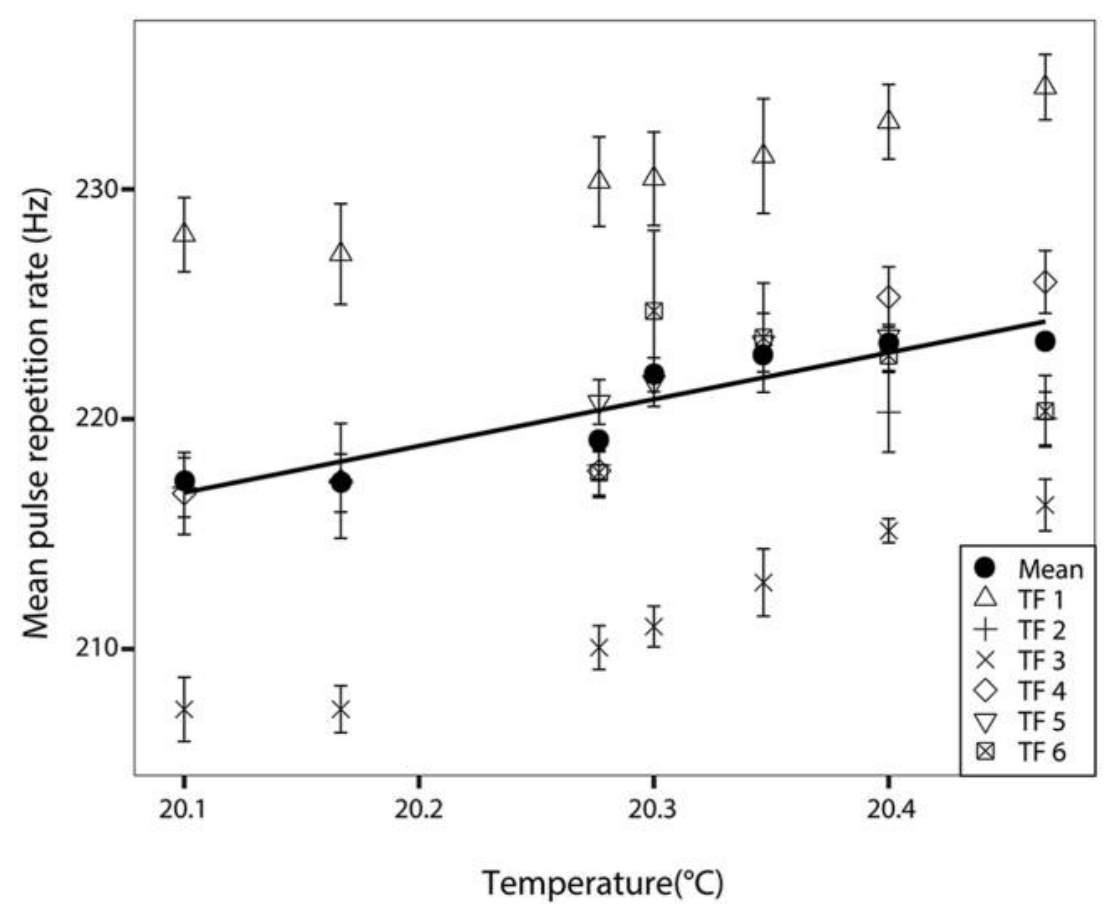

Figure 6. Effect of temperature on individual pulse repetition rate (PRR). The mean PRR of toadfish $(n=6)$ is plotted versus water temperature $\left({ }^{\circ} \mathrm{C}\right)$ over a 5-hour period from 2045 July 8 to 0145 July 9,2016 . PRR for each fish was pooled with mean $\pm 1 \mathrm{SD}$ for each fish. The black solid circles represent the mean PRR for all fish at each temperature. The solid line is a linear regression $(y=20.32 x-191.30)$. TF, toadfish. 


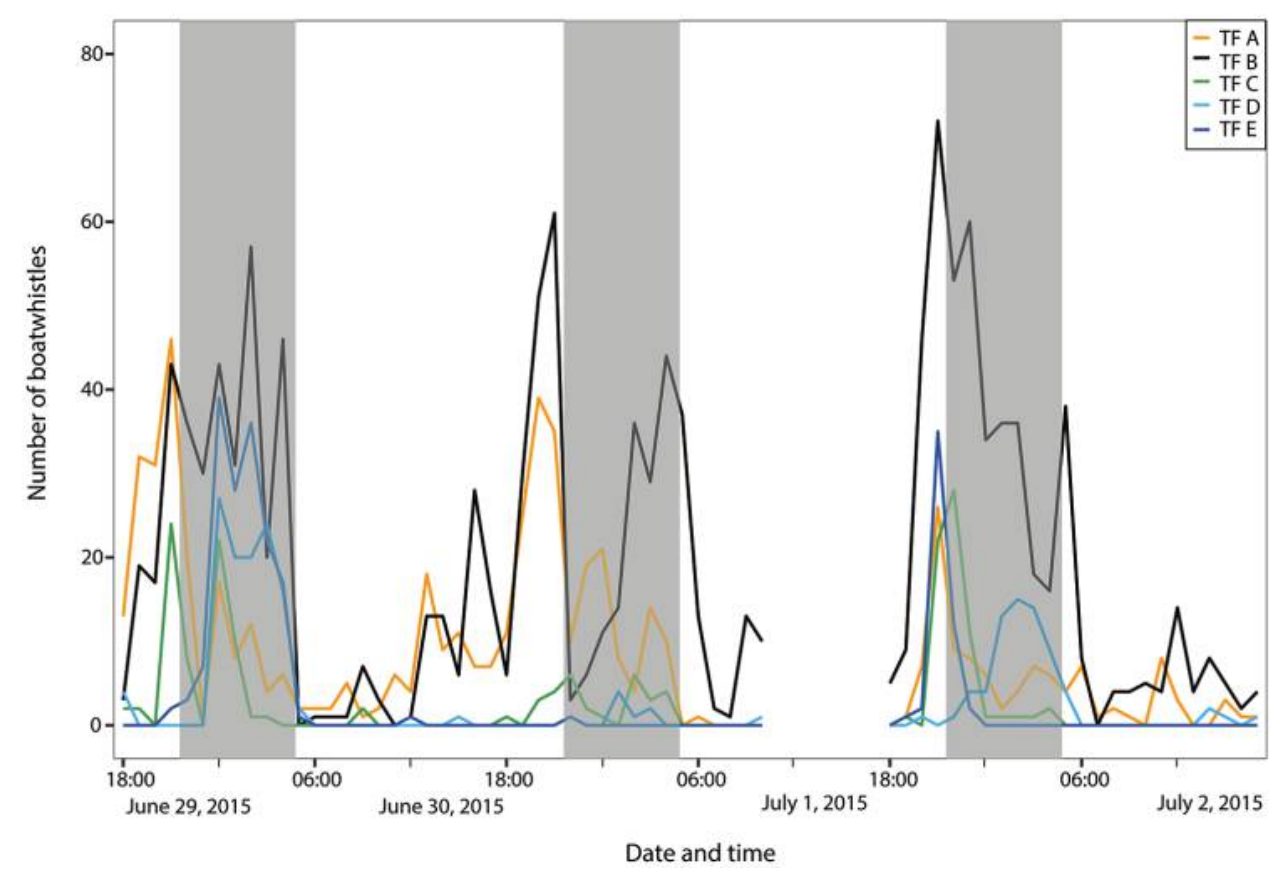

Figure 7. Individual variation in calling rate. The total number of boatwhistles for the first 10 minutes of each hour for 5 male toadfish is tracked between 1800 June 29 and 1700 July 2, 2015. Shaded gray boxes represent the time between sunset and sunrise, which was about 2020 and 0512 , respectively, during this time period. TF, toadfish.

Edds-Walton et al., 2002). Passive acoustic studies that monitored oyster toadfish populations over longer periods of time were also unable to track individuals (Monczak et al., 2017; Ricci et al., 2017). Although a single hydrophone location precludes the ability to determine the location of individual toadfish, male toadfish show high site fidelity (Gray and Winn, 1961; Putland et al., 2018) during the breeding season; and it is highly probable that vocalizations were recorded from the same fish during the breeding season. Short-term variation in acoustic signatures and amplitudes was minimal, which allowed individual toadfish to be tracked over extended time periods (72 hours). However, the SoundTrap needed frequent removal for battery charging and data download; and while many of these changeovers were accomplished in a few hours during relative lull in calling in the afternoon, complications or adverse weather conditions contributed to more extended lapses in coverage. Thus, these interruptions, combined with rapidly changing boatwhistle properties and the ever-evolving soundscape of Eel Pond, made it difficult to track the same individuals past 72 hours with the same degree of confidence.

\section{Seasonal variation}

The SoundTrap deployment successfully bracketed the boatwhistle season, because advertisement calls were not detected until after 72 hours of in situ recording (initial water temperature $12.6^{\circ} \mathrm{C}$ ) and stopped 48 hours before seasonal hydrophone removal; this allowed resident calling rates and spectral properties to be tracked throughout the breeding season. Sound production is controlled by motor neurons innervating the sonic muscle of the swim bladder (Bass and Baker, 1991). The neuronal firing rates are temperature dependent, with higher firing rates at warmer temperatures, resulting in higher frequencies (pulse repetition rates) in the boatwhistle (Bass and Baker, 1991). Boatwhistles were not detected until water temperatures reached $14.5^{\circ} \mathrm{C}$, which is consistent with other studies that did not detect boatwhistles until water temperatures exceeded $15^{\circ} \mathrm{C}$ (Fine, 1978; Edds-Walton et al., 2002).

Early-season "precursor" boatwhistles in toadfish have been noted, but the waveforms have not been characterized or described (Fish, 1954; Gray and Winn, 1961; Fine, 1978). The initial boatwhistles were infrequent and short, with an indistinct broadband grunt and inconsistent periodicity in the second segment. It is not clear whether the pacemaker and/or the sonic muscle was incapable of generating sound at lower temperatures or whether other physiological processes prevent boatwhistle generation below $14{ }^{\circ} \mathrm{C}$. It has been hypothesized that the second, tonal segment of the boatwhistle is the main attractant for female fish (Edds-Walton et al., 2002; Mensinger, 2014), so the functional significance of these shorter calls with variable tonal segments is unclear. Fertilized nests in the Cape Cod, Massachusetts, area are often not detected until several weeks after call onset, coinciding with the longer, distinct, and more uniform tonal segments, suggesting that these early-season calls are not used for mate attraction. This is consistent with previous behavioral experiments that showed that short-duration calls $(75-150 \mathrm{~ms})$ with mixed tones and low PRRs $(<180 \mathrm{~Hz})$ suppress male response calls, indicating 
that these spectral properties are presumably less attractive (Winn, 1972). However, the early precursor vocalizations may serve as territorial advertisement or may induce reproductive behavior in females. Later-season calls decreased in duration but retained well-defined tonal segments. These calls all showed high PRR, and the shorter durations may be attributed to the inability of the sonic muscles to continue high contraction rates for longer-duration calls (Rome and Lindstedt, 1998).

Temperature appears to be a strong influence on PRR, because water temperature changes were closely related to PRR during both short-term individual tracking and long-term population monitoring. The close relationship between temperature and PRR suggests that much of the variation in PRR is linked to temperature. However, boatwhistle PRRs and reduced calling rates in the early and late mating season might be attributed to pre- and post-spawning factors, including hormonal levels, absence of gravid females (Gray and Winn, 1961; Lowe, 1975; Fine, 1978), or physiological condition. These patterns of temperature dependency must be taken with caution, because additional factors, internal (hormonal) and external (social, tidal, lunar, etc.), may also influence vocalization patterns (Amorim et al., 2011; Ladich, 2018).

\section{Daily variation}

Continuous recording allowed short-term monitoring of individual oyster toadfish. Although previous studies have monitored oyster toadfish calling across the entire mating season, they did not follow individual fish (Fine, 1978; Monczak et al., 2017; Ricci et al., 2017). The current study shows the relationship between ambient water temperature and PRR of individual toadfish over an extended time period (up to 36 hours). The relationship between boatwhistle parameters (e.g., PRR, duration) and male reproductive success needs further investigation; however, the recent development of a hydrophone array that can localize toadfish nest sites (Putland et al., 2018) might allow correlation of vocalization with reproductive success (i.e., eggs in nest). Toadfish sound production is closely linked to temperature, which may have implications for toadfish mating seasons in future ocean warming conditions, when the ocean temperatures are predicted to increase by $3-5{ }^{\circ} \mathrm{C}$ by the year 2100 (IPCC, 2014).

The current study suggests that male toadfish monitor conspecific vocalizations and alternate calls to avoid overlap, where less than $1 \%$ of the boatwhistles overlap with other callers. Complex acoustic behavior has been previously observed in oyster toadfish, when males produce grunts during conspecific boatwhistles to jam calls and alter their acoustic parameters (Mensinger, 2014). The large differences observed in individual toadfish vocalization rates might indicate a dominant acoustic hierarchy, with one or two fish monopolizing the soundscape. The changes in calling rates might also affect calling parameters, as shown in the Lusitanian toadfish, Halobatrachus didactylus (Amorim et al., 2011). Whether changes in calling rates lead to greater reproductive success remains to be determined, because nearby males might save energy by remaining quiet to allow the dominant males to attract the females to the area and then start vocalizing.

\section{Conclusions}

Continuous monitoring of the resident oyster toadfish population allowed a detailed characterization of the seasonal and daily calling patterns and lent further insight into these fishes' complex vocal interactions. Resident toadfish calling rates peaked in mid to late June $\left(\sim 19^{\circ} \mathrm{C}\right)$. Boatwhistle calls change in acoustic structure throughout the mating season, and PRR is related to water temperature in both the long term (months) and the short term (days). This study brings light to the toadfish acoustic repertoire and lays a foundation for improved monitoring of this resident population in future years.

\section{Acknowledgments}

We thank Emily Cardinal for help with data collection and initial hydrophone setup, the Marine Resources Center at Marine Biological Laboratory for dock space and resources, John Atkins for SoundTrap hydrophone support, and Beth Giuffrida for analysis support. Rosalyn Putland and Jenni Stanley are gratefully acknowledged for coding assistance. We also thank UCSB PSTAT for statistics guidance. We are also grateful to the three anonymous reviewers and the editor for their comments. This study was made possible by National Science Foundation grants IOS 1354745 and DBI 1359230.

\section{Literature Cited}

Amorim, M. C. P. 2006. Diversity of sound production. Pp. 71-105 in Communication in Fishes, F. Ladich, S. P. Collin, P. Moller, and B. G. Kapoor, eds. Science Publishers, Enfield, NH.

Amorim, M. C. P., and R. Vasconcelos. 2008. Variability in the mating calls of the Lusitanian toadfish Halobatrachus didactylus: cues for potential individual recognition. J. Fish Biol. 73: 1267-1283.

Amorim, M. C. P., R. Vasconcelos, J. Marques, and F. Alamada. 2006. Seasonal variation of sound production in the Lusitanian toadfish Halobatrachus didactylus. J. Fish Biol. 69: 1892-1899.

Amorim, M. C. P., J. M. Simões, V. C. Almada, and P. J. Fonseca. 2011. Stereotypy and variation of the mating call in the Lusitanian toadfish, Halobatrachus didactylus. Behav. Ecol. Sociobiol. 65: 707-716.

Bass, A. H., and R. Baker. 1991. Evolution of homologous vocal control traits. Brain Behav. Evol. 38: 240-254.

Bass, A. H., and J. R. McKibben. 2003. Neural mechanisms and behaviors for acoustic communication in teleost fish. Prog. Neurobiol. 69: 126.

Beecher, M. D. 1989. Signalling systems for individual recognition: an information theory approach. Anim. Behav. 38: 248-261.

Bioacoustics Research Program. 2014. Raven Pro: interactive sound analysis software (version 1.4). [Computer software]. Cornell Lab of Ornithology, Ithaca, NY. Available: http://www.birds.cornell.edu/raven [2015, June 20].

Edds-Walton, P. L., L. A. Mangiamele, and L. C. Rome. 2002. Variations of pulse repetition rate in boatwhistle sounds. Bioacoustics 13: 153-173. 
Fay, R. R., and P. L. Edds-Walton. 1997. Diversity in frequency response properties of saccular afferents of the toadfish, Opsanus tau. Hear. Res. 113: 235-246.

Fine, M. L. 1978. Seasonal and geographical variation of mating call of oyster toadfish Opsanus tau L. Oecologia 36: 45-57.

Fine, M. L. 1983. Frequency-response of the swimbladder of the oyster toadfish. Comp. Biochem. Physiol. A Physiol. 74: 659-663.

Fish, J. F. 1972. The effect of sound playback on the toadfish. Pp. 386434 in Behavior of Marine Animals, H. E. Winn and B. L. Olla, eds. Plenum Press, New York.

Fish, M. P. 1954. The character and significance of sound production among fishes of the western North Atlantic. Bull. Bingham Oceanogr. Collect. 14: 3-109.

Gray, G. A., and H. E. Winn. 1961. Reproductive ecology and sound production of the toadfish Opsanus tau. Ecology 28: 9.

IPCC (Intergovernmental Panel on Climate Change). 2014. Climate Change 2014: Mitigation of Climate Change. Working Group III Contribution to the Fifth Assessment Report of the Intergovernmental Panel on Climate Change. Cambridge University Press, Cambridge.

Ladich, F. 2018. Acoustic communication in fishes: Temperature plays a role. Fish Fish. 19: 598-612.

Lowe, T. P. 1975. Reproductive ecology of oyster toadfish (Opsanus tau) in Charlestown Pond, Rhode Island. Ph.D. dissertation, University of Rhode Island, Kingston.

Maruska, K. P., and A. F. Mensinger. 2009. Acoustic characteristics and variations in grunt vocalizations in the oyster toadfish Opsanus tau. Environ. Biol. Fish. 84: 325-337.

Maruska, K. P., and A. F. Mensinger. 2015. Directional sound sensitivity in utricular afferents in the toadfish Opsanus tau. J. Exp. Biol. 218: 17591766.

MathWorks. 2018. MATLAB: the language of technical computing: computation, visualization, programming. [Computer software]. MathWorks, Natwick, MA. Available: https://www.mathworks.com/products/matlab .html [2018, July 10].

Mensinger, A. F. 2014. Disruptive communication: stealth signaling in the toadfish. J. Exp. Biol. 217: 344-350.

Mensinger, A. F., N. N. Price, H. E. Richmond, J. W. Forsythe, and R. T. Hanlon. 2003. Mariculture of the oyster toadfish: juvenile growth and survival. N. Am. J. Aquac. 65: 289-299.
Monczak, A., A. Berry, C. Kehrer, and E. W. Montie. 2017. Long-term acoustic monitoring of fish calling provides baseline estimates of reproductive time-lines in the May River estuary, southeastern USA. Mar. Ecol. Prog. Ser. 581: 1-19.

NWS (National Weather Service). 2015. NWS Boston - sunrise and sunset/moonrise and moonset. [Online]. National Oceanic and Atmospheric Administration, Washington, DC. Available: https://www.weather.gov /box/sunmoon [2016, July 22].

Putland, R. L., A. G. Mackiewicz, and A. F. Mensinger. 2018. Localizing individual soniferous fish using passive acoustic monitoring. Ecol. Inform. 48: 60-68.

Radford, C. A., and A. F. Mensinger. 2014. Anterior lateral line nerve encoding to tones and play-back vocalisations in free-swimming oyster toadfish, Opsanus tau. J. Exp. Biol. 217: 1570-1579.

Ricci, S. W., D. W. R. Bohnenstiehl, D. B. Eggleston, M. L. Kellogg, and R. P. Lyon. 2017. Oyster toadfish (Opsanus tau) boatwhistle call detection and patterns within a large-scale oyster restoration site. PLoS One 12: $\mathrm{e} 0182757$.

Rome, L. C., and S. L. Lindstedt. 1998. The quest for speed: muscles built for high-frequency contractions. Physiology 13: 261-268.

RStudio Team. 2018. RStudio: integrated development for R. [Computer software]. RStudio, Boston. Available: http://www.rstudio.com/ [2018, June 5].

Skoglund, C. R. 1961. Functional analysis of swimbladder muscles engaged in sound production of the toadfish. J. Biophvs. Biochem. Cvtol. 10: 187200.

Tavolga, W. N. 1958. Underwater sounds produced by two species of toadfish, Opsanus tau and Opsanus beta. Bull. Mar. Sci. Gulf Caribb. 8: 278285.

Tides and Currents. 2015. Woods Hole, MA - station ID: 8447930. [Online]. National Oceanic and Atmospheric Administration, Washington, DC. Available: https://tidesandcurrents.noaa.gov/stationhome.html?id $=8447930$ [2015, August 10].

Wilkins, M. R., N. Seddon, and R. J. Safran. 2013. Evolutionary divergence in acoustic signals: causes and consequences. Trends Ecol. Evol. 28: $156-166$.

Winn, H. E. 1972. Acoustic discrimination by the toadfish with comments on signal systems. Pp. 361-385 in Behavior of Marine Animals, Vol. 2, Vertebrates, H. E. Winn and B. Olla, eds. Plenum Press, New York. 


\section{Appendix}

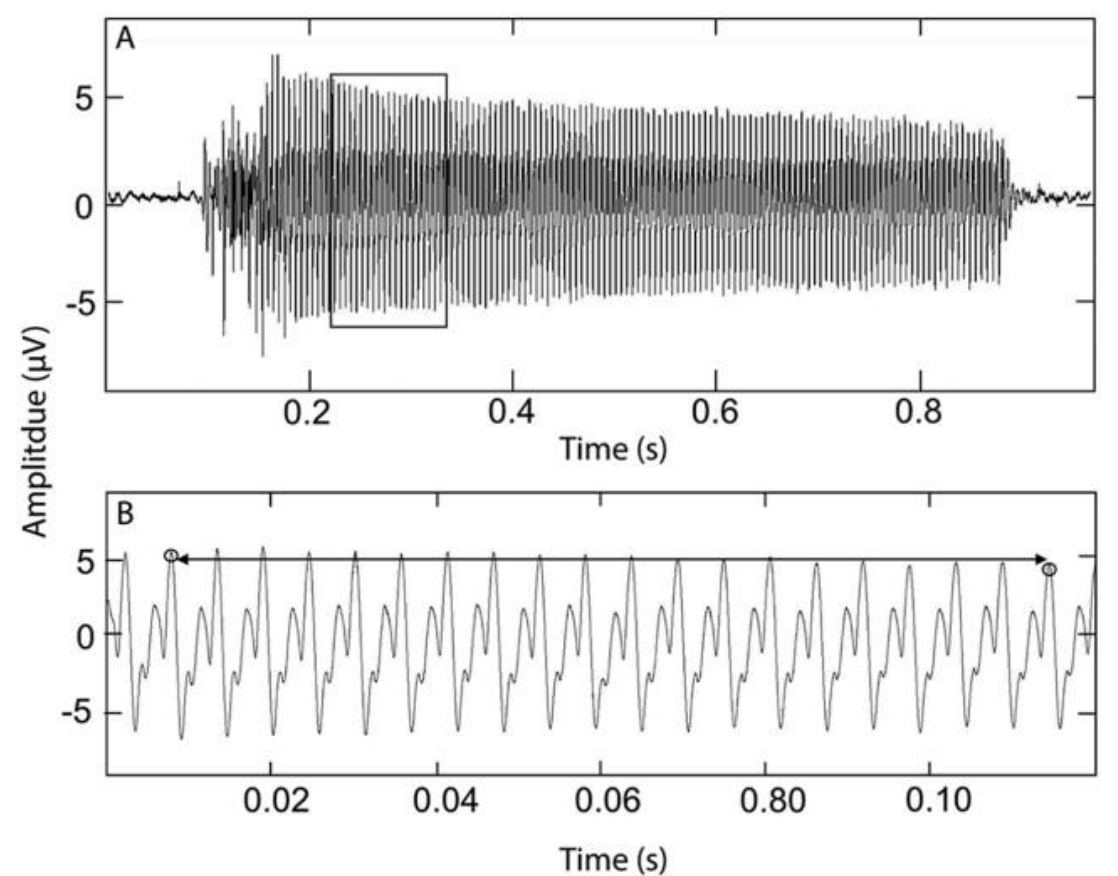

Figure A1. Manual boatwhistle pulse repetition rate (PRR) calculation. (A) Full-waveform view of a boatwhistle with a portion of the second, tonal segment boxed for PRR calculation. (B) Zoomed-in view; the first 20 contiguous pulses with approximately equal amplitudes of the second, tonal segment (black arrows) are selected and divided as $\mathrm{PRR}=$ number of pulses/time. Here, PRR $=20$ pulses $/ 0.112 \mathrm{~s}=$ $178.6 \mathrm{~Hz}$. 